\title{
Technical note: Simulation of dose build-up in proton pencil beams
}

\author{
Laurent Kelleter, ${ }^{1, *}$ Benjamin Zhen-Hong Tham,${ }^{2}$ Ruben Saakyan, ${ }^{1}$ \\ Jennifer Griffiths, ${ }^{2}$ Richard Amos, ${ }^{2}$ Simon Jolly, ${ }^{1}$ and Adam Gibson ${ }^{2}{ }^{\dagger}$ \\ ${ }^{1}$ Dept. Physics and Astronomy, University College London, Gower Street, WC1E 6BT London, UK \\ ${ }^{2}$ Dept. Medical Physics and Biomedical Engineering, \\ University College London, Gower Street, WC1E 6BT London, UK
}

(Dated: June 15, 2019)

\begin{abstract}
Purpose: The purpose of this study is to characterise the magnitude and depth of dose build-up in pencil beam scanning proton therapy.

Methods: We simulate the integrated depth-dose curve of realistic proton pencil beams in a water phantom using the the Geant4 Monte Carlo toolkit. We independently characterise the electronic and protonic components of dose build-up as a function of proton beam energy from 40 to $400 \mathrm{MeV}$, both with and without an air gap.

Results: At clinical energies, electronic build-up over a distance of about $1 \mathrm{~mm}$ leads to a dose reduction at depth of the basal layer $(0.07 \mathrm{~mm})$ by up to $6 \%$ compared to if no build-up effect were present. Protonic build-up reduces the dose to the basal layer by up to $16 \%$ and has effects at depths of up to $150 \mathrm{~mm}$. Secondary particles with a mass number $A>1$ do not contribute to dose build-up. An air gap of one metre has no significant effect on protonic build-up but reduces electronic build-up below $1 \%$.

Conclusions: Protonic and electronic dose build-up are relevant for accurate dosimetry in proton therapy although a realistic air gap reduces the electronic build-up to levels where it can be safely neglected. We recommend including electrons and secondary protons in Monte Carlo-based treatment planning systems down to a predicted range of $10-20 \mu \mathrm{m}$ in order to accurately model the dose at depths of the basal layer, no matter the size of the air gap between nozzle and patient.
\end{abstract}

Keywords: proton therapy, dose build-up, secondary particles, skin sparing

\section{INTRODUCTION}

The build-up effect in proton therapy, whereby the skin dose is lower than expected and "builds up" over a finite distance, is substantially under-researched compared to that in photon therapy. Early investigations demonstrated that for $185 \mathrm{MeV}$ protons, the surface dose was about $7 \%$ lower than the dose that would have been expected in the absence of build-up ${ }^{1}$. This and other work ${ }^{2-4}$ differentiated between electronic build-up, which occurs over a depth of approximately $1.5 \mathrm{~mm}$ in water, and protonic build-up to a depth of about $40 \mathrm{~mm}$ in water. They noted that both electron and protonic build-up are sometimes not observed, particularly at low beam energies ${ }^{4}$. Recent work by Pfuhl et al. ${ }^{5}$ provided high-precision measurements and Fluka simulations of the build-up at a single energy and claimed that heavy target fragments with $A>1$ contribute to the dose build-up.

Treatment planning systems for proton therapy such as Eclipse (Varian) ${ }^{6}$, Pinnacle $^{3}\left(\text { Philips) }{ }^{7}, \text { XiO (Elekta) }\right)^{8}$ and RayStation (RaySearch Laboratories) $)^{9}$ tend to deal with build-up using configurable, empirical correction factors which lead to uncertainties in the build-up region. Such uncertainties would be reduced with Monte Carlo-based treatment planning. This is currently computationally prohibitively expensive but could be sped up by simplifying the physics model so that, for example, only certain particle species are tracked, or particles are neglected after their energy falls below a threshold. One aim of this paper is to inform the discussion about the requirements for accurate modelling of the build-up region.

Experimental measurements of the build-up effect are challenging as they require measurements very close to the surface to be made with a depth resolution $<<1 \mathrm{~mm}$ in tissue- or water-equivalent media. Furthermore, it is very difficult to measure the relative contribution of different particles comprising the build-up experimentally. As such, detailed simulations are necessary to adequately study the build-up process. This is the first paper to thoroughly quantify the build-up region in proton therapy with simulations over the full range of clinically relevant beam energies.

* laurent.kelleter@ucl.ac.uk

$\dagger$ adam.gibson@ucl.ac.uk 


\section{METHOD}

\section{A. Geant4 simulation settings}

In this simulation study, the Monte Carlo software platform Geant4 v10.3.2 was used with the standard electromagnetic physics list with a binary cascade model for non-elastic scattering, as recommended for proton therapy ${ }^{10}$. The OPT3 and OPT4 constructors were tested but no difference was found in the build-up region. We selected OPT3 in this work. Production thresholds (range cuts) affect the production of secondary electrons, positrons and photons in Geant4. If the predicted range of a secondary particle is lower than the range cut, no secondary particle is produced and the energy loss is attributed to the parent particle. Therefore, the range cut has a strong influence on the size and shape of the energy deposition curves of secondary protons and electrons. We quote the dose at the first radio-sensitive layer of the skin, the basal layer, at a depth of $0.07 \mathrm{~mm}$ and so chose a range cut of $10 \mu \mathrm{m}$. To ensure that each tracked particle performs at least two steps before coming to rest, we apply a step maximum of $5 \mu \mathrm{m}$. $10^{6}$ protons were launched per simulation. The dose is scored within the Geant4 class G4SteppingAction and is split up into contributions from different particle species. The scoring bin size is $5 \mu \mathrm{m}$, equal to the maximum step size. Secondary protons are are differentiated from primary protons using the Track ID which is 1 for primary particles and $>1$ otherwise.

The simulation geometry consists of a $1 \times 1 \times 1 \mathrm{~m}^{3}$ water phantom. This ensures that the depth is large enough that all primary protons are stopped in the phantom and that the transverse area is large enough that all charged secondary particles, including those that are scattered outside the main beam are included in simulations of the integral depthdose curve. The water volume sits within a world volume that contains air at Geant 4 standard conditions. The ionisation energy of water is set to be $78 \mathrm{eV}^{11}$. We investigate proton energies $E$ ranging from 40 to $400 \mathrm{MeV}$. Each proton beam has a Gaussian energy spectrum with a mean of $E$ and a width of $\sigma_{E}=0.01 \times E$. The pencil beam has a realistic Gaussian spatial profile with a width of $\sigma_{R}=4 \mathrm{~mm}$ and an angular spread of $3.2 \mathrm{mrad}$, which are typical values for clinical beams ${ }^{12}$. The proton source was placed in contact with the centre of one face of the water volume other than when an air gap was introduced.

\section{B. Definition of dose build-up}

Fig 1 shows the integrated depth-dose curve (Bragg curve) of a $200 \mathrm{MeV}$ proton beam with the depth-dose curves of secondary particles created along the path. All particles heavier than protons (mass number $A>1$ ) are referred to as heavy ions. A dose build-up effect is visible in the depth-dose curves of electrons and secondary protons.

We define the magnitude of dose build-up for proton pencil beams as the difference between the dose deposited at two reference depths (shallow and deep), quoted from the secondary particle depth-dose curves and normalised to the total dose at the deep reference depth. The maximum of the secondary proton depth-dose curve scales with the beam energy and is located at approximately at half of the beam range. Thus, we define the deep reference depth of the protonic build-up $\beta_{p}$ to be $d_{p, \text { deep }}=0.5 R_{0}$. The range of the proton beam is defined as the point after the Bragg peak at which the dose decreases to $80 \%$ of its maximum value: $R_{0}=d_{80}$. The minimum depth that always includes the whole of the electronic build-up $\beta_{e}$ was found empirically and is $0.01 R_{0}$. Therefore, we choose the deep reference depth of the electronic build-up to be $d_{e, \text { deep }}=0.01 R_{0}$. The lower reference depth for both build-up metrics is chosen to be the basal layer of the skin $d_{\text {shallow }}=0.07 \mathrm{~mm}^{13}$, which is determined by fitting a straight line to the dose between $0.05 \mathrm{~mm}$ and $0.09 \mathrm{~mm}$ depth and evaluating the fit function at $0.07 \mathrm{~mm}$. The uncertainty on the dose is calculated from the uncertainties on the fit parameters by using Gaussian error propagation. The dose at the deep reference depths is determined by taking the average dose in an interval of $d_{\text {deep }} \pm 0.05 d_{\text {deep }}$ on either side of the deep reference depth.

The full definitions of the protonic build-up $\beta_{p}$ and electronic build-up magnitudes $\beta_{e}$ are as follows:

$$
\begin{aligned}
& \beta_{p}\left(d_{p, \text { deep }}\left(R_{0}\right), d_{\text {shallow }}\right)=\frac{D_{p}\left(d_{p, \text { deep }}\left(R_{0}\right)\right)-D_{p}\left(d_{\text {shallow }}\right)}{D_{\text {tot }}\left(d_{p, \text { deep }}\left(R_{0}\right)\right)}=\frac{D_{p}\left(0.5 R_{0}\right)-D_{p}(0.07 \mathrm{~mm})}{D_{\text {tot }}\left(0.5 R_{0}\right)} \\
& \beta_{e}\left(d_{e, \text { deep }}\left(R_{0}\right), d_{\text {shallow }}\right)=\frac{D_{e}\left(d_{e, \text { deep }}\left(R_{0}\right)\right)-D_{e}\left(d_{\text {shallow }}\right)}{D_{\text {tot }}\left(d_{e, \text { deep }}\left(R_{0}\right)\right)}=\frac{D_{e}\left(0.01 R_{0}\right)-D_{e}(0.07 \mathrm{~mm})}{D_{\text {tot }}\left(0.01 R_{0}\right)}
\end{aligned}
$$

where $D_{t o t}(x)$ describes the total depth-dose curve and $D_{e}(x)$ and $D_{p}(x)$ refer to the depth-dose curves of electrons and secondary protons, respectively. This definition allows to interpret metrics $\beta_{p}$ and $\beta_{e}$ as the amount of "missing" dose at the basal layer due to dose build-up. 
We define the $95 \%$ build-up depths $d_{e, 95}$ and $d_{p, 95}$ as the depths at which the electronic and protonic build-up magnitude reach $95 \%$ of their final values, respectively. The build-up depth is determined by evaluating a fitted line in an area around the expected build-up depth $( \pm 20 \%$ on either side). The statistical uncertainty of the build-up depth is calculated by Gaussian error propagation from the uncertainties on the fit parameters. The mathematical definitions of $d_{95}$ are:

$$
\begin{aligned}
& d_{p, 95}=d \text { such that } \beta_{p}\left(d, d_{\text {shallow }}\right)=0.95 \times \beta_{p}\left(d_{p, \text { deep }}\left(R_{0}\right), d_{\text {shallow }}\right) \\
& d_{e, 95}=d \text { such that } \beta_{e}\left(d, d_{\text {shallow }}\right)=0.95 \times \beta_{e}\left(d_{e, \text { deep }}\left(R_{0}\right), d_{\text {shallow }}\right)
\end{aligned}
$$

\section{RESULTS}

\section{A. Dose build-up as a function of energy}

The simulated depth-dose curves for secondary protons (Fig 1: finely dashed red line) and heavy ions (dotted gray line) are in accordance with simulations published by Paganetti ${ }^{14}$, although the depth-dose for electrons (coarsely dashed blue line) with ranges as low as $10 \mu \mathrm{m}$ is reported for the first time. The depth-dose curves of both electrons and secondary protons show a dose build-up at the entrance to the water phantom. The effect of electronic build-up can be seen from $0-0.5 \mathrm{~mm}$ in the electron depth-dose curve and that of protonic build-up stems from secondary protons from $0-100 \mathrm{~mm}$. In contrast to what was found by Pfuhl et al. ${ }^{5}$ we do not see evidence that particles with $A>1$ contribute to the build-up effect on the short or the long length scale.

Proton beams with energies from 40 to $400 \mathrm{MeV}$ were simulated in order to cover the full range of clinically relevant proton beam energies and go beyond them because of their relevance for potential future proton radiography and proton CT applications. Both electronic and protonic build-up increase monotonically with beam energy and reach values of $6 \%$ and $16 \%$ at a beam energy of $250 \mathrm{MeV}$, respectively (Fig 2a). The statistical uncertainty results in error bars that are smaller than the size of the marker points.

The most important simulation parameter for the dose build-up is the choice of the Geant4 range cut. The range cut needs to be smaller than $20 \mu \mathrm{m}$ to ensure that the dose at the basal layer is robust. Furthermore, the protonic build-up decreases from $11.5 \%$ to $11.0 \%$ for a $200 \mathrm{MeV}$ proton beam when calculated with a range cut of $20 \mu \mathrm{m}$ compared to $10 \mu \mathrm{m}$. The shorter range cut ensures that electrons with ranges between 10 and $20 \mu \mathrm{m}$ are tracked and so contribute to the electron dose instead of to the secondary proton dose.

Both the electronic and the protonic $95 \%$ build-up depths increase approximately linearly with beam energy (Fig 2b) as the mean momentum transfer to secondary particles increases.

\section{B. Effect of an air gap on dose build-up}

In a realistic clinical setting there is an air gap of up to one metre between the beam nozzle and the patient which we simulate using a $200 \mathrm{MeV}$ proton beam (Fig 2c). The electronic build-up magnitude decreases exponentially as the beam passes through air from a maximum of $5 \%$ with no air gap to below $2 \%$ when the air gap exceeds $30 \mathrm{~cm}$. The protonic build-up magnitude however remains largely unaffected of an air gap of up to $1 \mathrm{~m}$.

\section{DISCUSSION}

We introduce robust definitions of dose build-up magnitude and depth. Fig 2a,b is intended to be used as a look-up table to researchers and clinicians to provide an estimate of the extent of the build-up effect for a specific proton pencil beam. Our work is the first to analyse the beam-energy dependence of dose build-up in proton therapy. We characterise the extent of electronic and protonic build-up, but find no evidence for build-up due to particles with mass number $A>1$.

Protonic build-up increases linearly from $0 \%$ at $40 \mathrm{MeV}$ to $16 \%$ at $250 \mathrm{MeV}$ and extends to a maximum depth of $150 \mathrm{~mm}$. Electronic build-up increases from $0 \%$ at $40 \mathrm{MeV}$ to $6 \%$ at $250 \mathrm{MeV}$ and occurs within $1 \mathrm{~mm}$ of the surface. Both lead to a modest skin-sparing effect. The electronic build-up depths agree with those in the literature ${ }^{2}$, but the magnitude and depth of protonic build-up are greater than those reported previously. Previously, protonic build-up has been measured relative to the total depth-dose curves and overlaps with the primary proton dose. The full extent of the secondary proton dose build-up is only revealed by the secondary proton depth-dose curve. 
In order to accurately model the dose build-up region in Monte Carlo Treatment Planning Systems it is necessary to include secondary protons and electrons in the simulation. High-LET particles with $A>1$ have little effect on dose build-up, but it is necessary to include electrons with an expected range as low as 10-20 $\mu \mathrm{m}$ in order to accurately predict the dose at the depth of the basal layer.

Electronic build-up depends strongly on size of an air gap. Although its magnitude might be negligible in a clinical setting with an air gap exceeding $50 \mathrm{~cm}$, always including electrons in Monte Carlo treatment planning would help to decrease dose uncertainties at the basal layer. The majority of the protonic build-up however, will always occur within the patient because it reaches its maximum only at half of the predicted proton range. Furthermore, dose monitors and beam degraders will perturb the primary proton beam and secondary protons generated upstream from the patient in a way which will depend on their position relative to the patient, their water-equivalent thickness and their material composition. Precise knowledge of the build-up effect in a specific treatment centre therefore requires accurate modelling of the beam line and the beam exit nozzle.

Simulations of realistic clinical beams, dose monitors and beam degraders will be part of future work as will an analysis of boundaries between different tissue types where a new secondary particle equilibrium needs to build up. In this work, we focused on the one-dimensional integrated depth-dose curve since beam divergence is shown to have very little influence on the entrance dose ${ }^{15}$. Further work will include the simulation of a three-dimensional patient anatomy. The increased build-up observed at higher energies may be beneficial for future high-energy proton delivery, such as may be used in proton CT. In addition, we encourage radiobiological studies to investigate the impact of high-LET particles on skin tissue due to the apparent lack of skin sparing for particles with $A>1$.

\section{CONCLUSION}

At clinically relevant energies $(40-250 \mathrm{MeV})$ and with a realistic air gap $(\sim 50 \mathrm{~cm})$ between beam nozzle and the absorbing medium, the protonic build-up reaches a maximum value of $16 \%$ of the entrance dose at $250 \mathrm{MeV}$ beam energy while the electronic dose build-up is found to be below $1 \%$ and therefore largely negligible. However,the magnitude of electronic dose build-up depends strongly on the size of the air gap. We therefore recommend to include electrons and secondary protons in Monte Carlo treatment planning systems down to a predicted range of 10-20 $\mu \mathrm{m}$ in order to accurately model the dose build-up at depths of the basal layer $(70 \mu \mathrm{m})$. Secondary particles with $A>1$ are found not to contribute to the dose build-up behind the basal layer.

\section{ACKNOWLEDGMENTS}

The authors are grateful to Prof. Bernard Gottschalk, Jailan Alshaikhi and the participants of the 2017 PPRIG workshop who provided valuable advice. SJ and RS acknowledge funding from STFC including grant ST/N000285/1. This project has received funding from the European Union's Horizon 2020 research and innovation programme under the Marie Sklodowska-Curie grant agreement No 675265.

\section{CONFLICT OF INTEREST STATEMENT}

The authors have no relevant conflicts of interest to disclose.

\section{REFERENCES}

[1] C. A. Carlsson and G. A. Carlsson. Proton dosimetry with $185 \mathrm{MeV}$ protons. Health Physics, 33(5):481-484, 1977.

[2] Harald Paganetti. Proton Therapy Physics. Medical Physics and Biomedical Engineering. CRC Press, 2012.

[3] Bernard Gottschalk, Ethan W. Cascio, Juliane Daartz, and Miles S. Wagner. On the nuclear halo of a proton pencil beam stopping in water. Phys. Med. Biol., 60(14):5627, 2015.

[4] Wayne D. Newhauser and Rui Zhang. The physics of proton therapy. Phys. Med. Biol., 60(8):R155, 2015.

[5] T Pfuhl, F Horst, C Schuy, and U Weber. Dose build-up effects induced by delta electrons and target fragments in proton Bragg curves - measurements and simulations. Phys. Med. Biol., 63, 2018. 
[6] Varian. Eclipse Proton Algorithms Reference Guide. 2015.

[7] Philips Medical Systems. Pinnacle ${ }^{3}$ IMPT/Spot Scanning Proton Treatment Planning Prototype User Manual. 2013.

[8] Elekta CMS Software. XiO Proton Beam Scanning System. 2010.

[9] RaySearch Laboratories AB. RayStation 4.7 Reference Manual. 2014.

[10] Andreas Resch, Alessio Elia, Hermann Fuchs, Antonio Carlino, Markus Stock, Dietmar Georg, and Loic Grevillot. Evaluation of electromagnetic and nuclear scattering models in GATE/Geant4 for proton therapy. Medical Physics, $46,2019$.

[11] Y Kumazaki, T Akagi, T Yanou, Suga D, Hishikawa Y, and T Teshima. Determination of the mean excitation energy of water from proton beam ranges. Radiation Measurements, 42, 2007.

[12] Clemens Grassberger and Harald Paganetti. Elevated let components in clinical proton beams. 56, 2011.

[13] ICRP. Recommendations of the International Commission on Radiological Protection. Pergamon Press, Oxford, UK, 2007.

[14] Harald Paganetti. Nuclear interactions in proton therapy: dose and relative biological effect distributions originating from primary and secondary particles. Physics in Medicine and Biology, 47, 2002.

[15] Harald Paganetti. Proton Beam Therapy, page 55. 2399-2891. IOP Publishing, 2017.

\section{FIGURE CAPTIONS}

Figure 1: Simulation of an integrated depth-dose curve in water of a $200 \mathrm{MeV}$ proton beam.

Figure 2: Evolution with beam energy (a,b) and air gap thickness at $200 \mathrm{MeV}$ beam energy (c) of electronic and protonic build-up magnitude and $95 \%$ build-up depth.

a) Build-up magnitude.

b) $95 \%$ build-up depth.

c) Build-up magnitude with air gap.

FIGURES

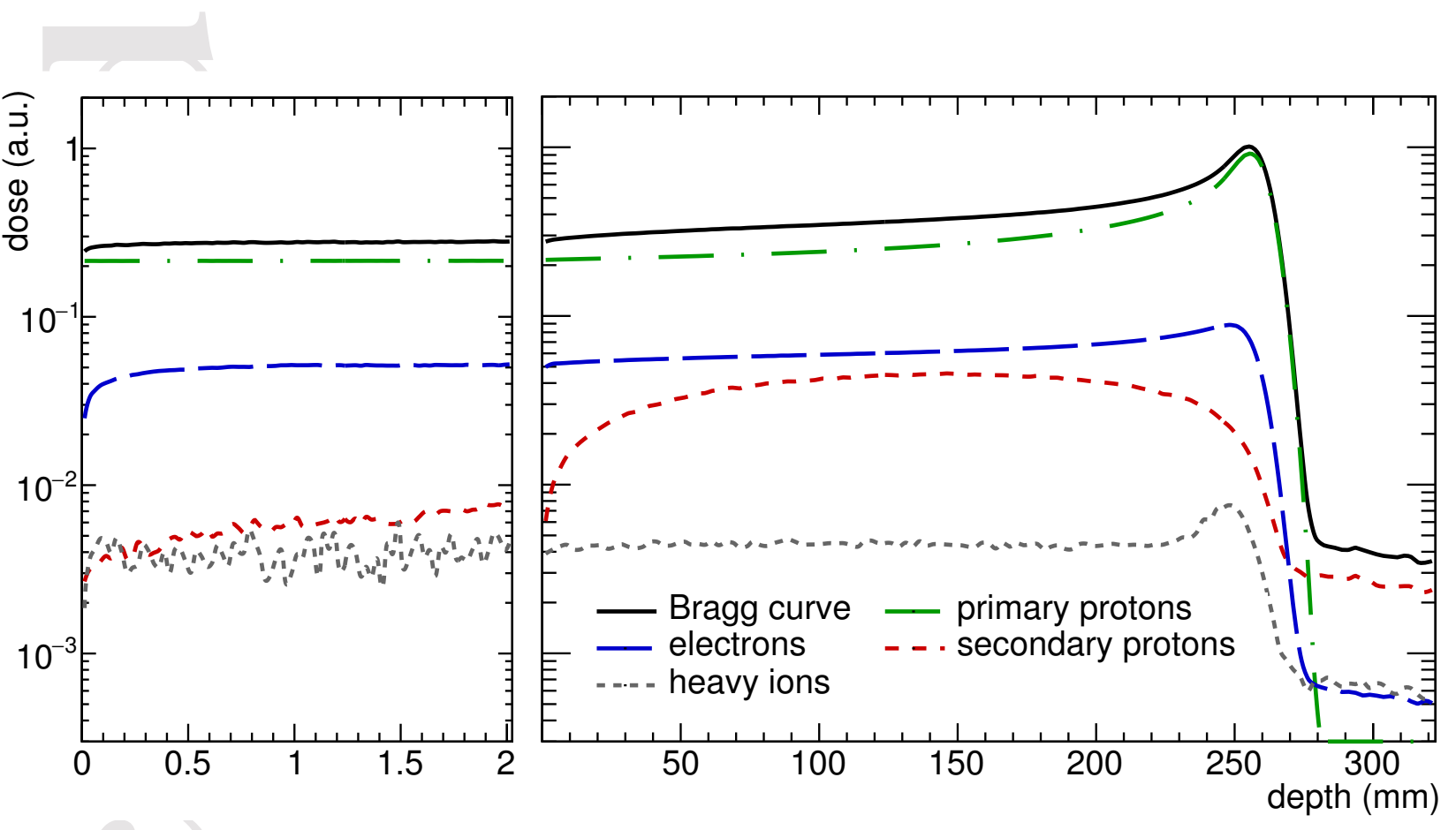

FIG. 1. Simulation of an integrated depth-dose curve in water of a $200 \mathrm{MeV}$ proton beam. 


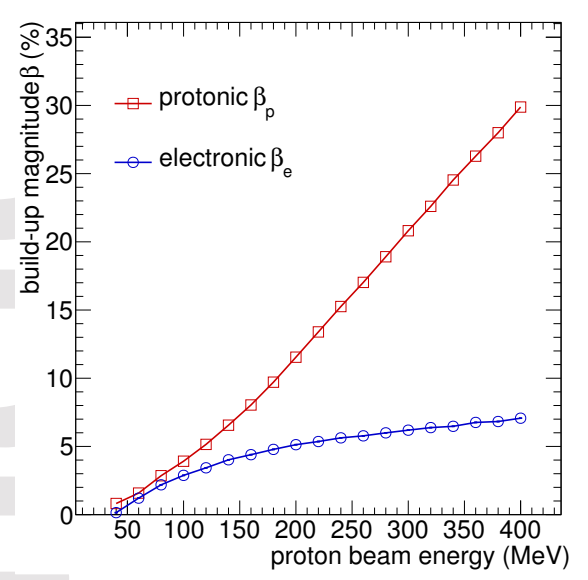

a) Build-up magnitude.

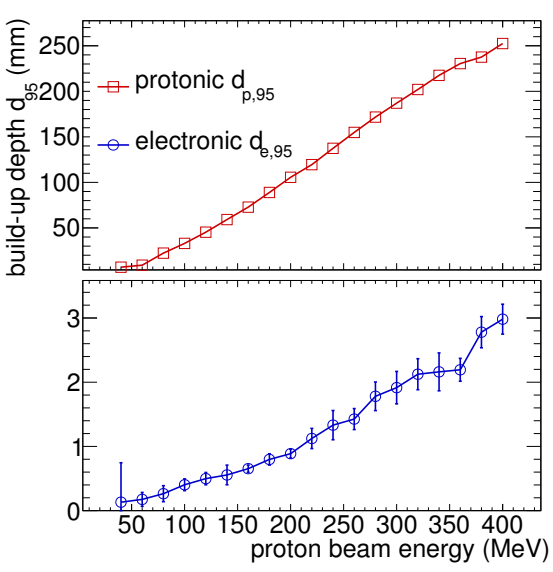

b) $95 \%$ build-up depth.

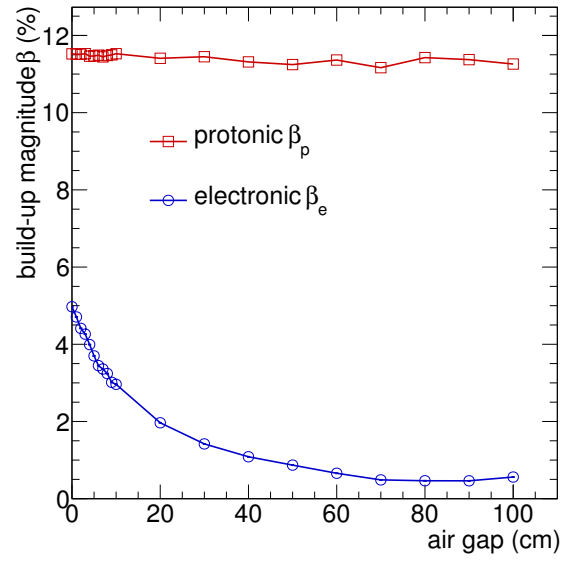

c) Build-up magnitude with air gap.

FIG. 2. Evolution with beam energy (a,b) and air gap thickness at $200 \mathrm{MeV}$ beam energy (c) of electronic and protonic build-up magnitude and $95 \%$ build-up depth. 